\title{
Forgiven the Right Way: The Role of Regulatory Fit in Brand Apologies and Forgiveness
}

\author{
Young-A Song, So Young Lee, Tae Rang Choi, and Marcos Duran
}

\begin{abstract}
In brand transgression context, some consumers forgive the brand because they want to gain the relationship back while others might forgive the brand because they do not want to lose the relationship. Using a regulatory fit perspective (Avnet and Higgins 2006), this research investigates how a match of motivational approach between consumers' goal orientation and brand apology impacts on brand forgiveness. The results show that consumers with a dominant promotion focus are more likely to forgive a brand when they receive a promotion-focused brand apology, while those with a dominant prevention focus are more likely to forgive a brand when receiving a prevention-focused brand apology.
\end{abstract}

Y.-A. Song $(\bowtie) \bullet$ S.Y. Lee • T.R. Choi $\bullet$ M. Duran

The University of Texas at Austin, Austin, TX, USA

e-mail: song.younga@utexas.edu; leesy8733@utexas.edu; sy.t.choi@utexas.edu;

marcosbhd@gmail.com 